\title{
Estudio Multicéntrico sobre Actitudes y Normas Culturales frente a la Violencia (proyecto ACTIVA): metodología
}

\author{
Marco Fournier, ${ }^{1}$ Rebecca de los Ríos, ${ }^{2}$ Pamela Orpinas ${ }^{3}$ \\ y Leandro Piquet-Carneiro ${ }^{4}$
}

RESUMEN El propósito de este artículo es describir las bases conceptuales y los métodos empleados en el estudio multicéntrico ACTIVA, así como el proceso que se cumplió en las diferentes etapas de la investigación. Se brinda información general sobre el enfoque, diseño y métodos que se aplicaron en las ciudades que participaron en el estudio: San Salvador, El Salvador; San José, Costa Rica; Cali, Colombia; Caracas, Venezuela; Salvador de Bahía y Río de Janeiro, Brasil; Santiago, Chile y Madrid, España. Los objetivos del estudio fueron: 1) analizar y comparar entre ciudades la prevalencia de conductas agresivas verbales y físicas hacia los niños, la pareja y personas que no son miembros de la familia; $y$ 2) identificar factores personales, ambientales $y$ socioeconómicos asociados con estas conductas violentas. El propósito del proyecto ACTIVA fue generar información que ayudara a orientar políticas y programas para la prevención de la violencia en las ciudades y brindar criterios para la toma de decisiones, así como recabar información que sirviera de base para evaluar políticas y programas de prevención. Para cumplir con los objetivos propuestos se procedió a elaborar y validar un cuestionario común, que se aplicó, mediante el método de entrevista cara-cara, a los residentes de hogares particulares urbanos entre los 18 y 70 años de edad. La muestra fue seleccionada en cada ciudad mediante un muestreo multietápico, estratificado y por conglomerados, y proporcionada según los estratos socioeconómicos y la densidad de la población y sin reemplazo o sustitución. La muestra final estuvo compuesta por 10821 personas. En general, los porcentajes de respuesta se encontraron comprendidos entre los límites establecidos, salvo algunas excepciones. En San José, Río de Janeiro y Madrid el tamaño de la muestra final fue ligeramente menor del esperado. Se registraron mayores rechazos en varones jóvenes adultos de los estratos altos, por lo cual en las muestras se observó una sobrerrepresentación de mujeres con predominio de los estratos medio y bajo. A manera de conclusiones se exponen los alcances y limitaciones, tanto del enfoque como de los métodos del estudio. Se destacan las aportaciones que brindan los estudios de este tipo cuando se efectúan análisis comparativos que permiten identificar las diferencias y semejanzas que se expresan en diferentes centros urbanos. Se indica que con el método propuesto es posible analizar la asociación entre las conductas violentas y diferentes factores de riesgo y atributos a nivel individual en un momento particular en el tiempo. Sin embargo, se advierte que este diseño tiene limitaciones a la hora de establecer relaciones causales y que no permitió evaluar el contexto en que se manifiesta y desarrolla la violencia en cada situación particular.

1 Instituto de Investigaciones Psicológicas, Universidad de Costa Rica, San José, Costa Rica. Dirección postal: Instituto de Investigaciones Psicológicas, Ciudad Universitaria Rodrigo Faio, San José, Costa Rica. Tel./Fax: (+506) 224-1844. Correo electrónico: mfournie@cariari.ucr.ac.cr
2 Asesora Regional de Investigaciones en Salud Pública, Coordinación de Investigaciones, División de Salud y Desarrollo Humano, Organización Panamericana de la Salud, Washington, D.C., Estados Unidos de América.
3 Universidad de Georgia, Departamento de Promoción de la Salud y Conductas Saludables, Athens, Georgia, EUA.

4 Departamento de Ciencias Políticas, Universidad de São Paulo, SP, Brasil. 
La violencia es reconocida como un obstáculo para el desarrollo de las naciones y una amenaza para la salud pública en la Región de las Américas (1). Para aliviar el problema de la agresión interpersonal y la lesión intencional se han elaborado y ejecutado diversos programas en la Región $(2,3)$. Sin embargo, es preciso profundizar en el conocimiento y la comprensión de la compleja trama de factores socioeconómicos, psicosociales y culturales que se asocian con la violencia y fundamentar un enfoque integrado de prevención. En 1996, la Organización Panamericana de la Salud (OPS) inició una investigación cooperativa y multinacional denominada proyecto ACTIVA, destinada a evaluar actitudes y normas culturales relacionadas con la violencia en ciudades de América Latina y España. Los objetivos de la investigación fueron: 1) analizar y comparar entre ciudades la prevalencia de conductas agresivas verbales y físicas hacia los niños, la pareja y personas que no son miembros de la familia, y 2) identificar factores personales, ambientales y socioeconómicos asociados con estas conductas violentas. El propósito general del proyecto ACTIVA fue generar información que ayudara a orientar políticas y programas para la prevención de la violencia en las ciudades y brindar criterios para la toma de decisiones, así como recabar información que sirviera como línea de base para evaluar políticas y programas de prevención.

El proyecto ACTIVA tuvo como referente inicial la teoría del aprendizaje cognitivo (4-6), que ha servido como base de diversos programas de promoción de la salud (7). En este estudio se partió de un primer modelo para entender los procesos que conducen o no a la violencia, el cual orientó la elección de los conceptos que se pretendía medir en esta investigación. Este modelo, basado en la teoría de Bandura sobre el comportamiento humano, fue desarrollado por el profesor Alfred McAlister (figura 1). Una descripción detallada del modelo se encuentra en el protocolo del proyecto ACTIVA (8). Las definiciones de los componentes centrales de este modelo se descri- ben en el cuadro 1. En el diagrama se muestra la función que desempeñan las actitudes y aptitudes tanto en el comportamiento violento como en el pacífico. En cada caso, el ambiente produce una situación a la cual responden el agresor y la víctima. Si la situación despierta una reacción emocional de enojo o frustración o una necesidad urgente de responder, la selección de respuestas conductuales se guiará por actitudes hacia tales respuestas y por su aceptabilidad social o moral (normas), así como por las expectativas de eficacia personal (capacidad) para actuar de forma agresiva o pacífica. El alcohol, las emociones, el estrés y otros factores pueden modificar el procesamiento de la información y los procesos de evaluación de la actitud, por ejemplo, al bajar los umbrales, limitar las opciones o impedir el razonamiento. Los factores ambientales, como la disponibilidad de armas y la privación económica, también influirán en los tipos de comportamiento que se elijan para hacer justicia o para autoprotegerse (8).

El modelo fue revisado por el grupo de los investigadores participantes, quienes analizaron sus ventajas y limitaciones. Este enfoque teórico, que representa una corriente de pensamiento dentro de las ciencias de la conducta, ofrece un análisis de las características y atributos de los individuos que son entendidos como factores protectores o de riesgo para la adopción de conductas y prácticas violentas o no violentas. Se reconoce que con este enfoque se pueden orientar intervenciones para modificar actitudes, desarrollar habilidades individuales en la resolución de conflictos interpersonales, controlar factores de riesgo y prevenir conductas agresivas. Aunque esto se valora como una ventaja del enfoque para intervenir a nivel individual, el enfoque presenta limitaciones para comprender y analizar la violencia como expresión social de desigualdades y de otro orden de conflictos y determinantes que trascienden el nivel psicosocial y el de los comportamientos de los individuos. Aunque este modelo orientó el estudio en sus grandes componentes, fue ampliado, adaptado e incluso reinterpretado por los investigadores. Una muestra de ello se puede apreciar en los artículos que integran esta monografía.

El propósito de este artículo es describir los métodos empleados en el estudio multicéntrico ACTIVA, así como el proceso que articuló las diferentes etapas de la investigación. Se brinda información sobre el diseño, el desarrollo del instrumento y sus componentes finales, así como sobre el proceso de selección de la muestra y la recolección de datos en las ocho ciudades que participaron en América Latina y España.

\section{MATERIALES Y MÉTODOS}

El proyecto ACTIVA es un estudio transversal en el cual se utilizó un cuestionario común y el método de entrevista estructurada cara-cara a residentes de hogares particulares urbanos. Las entrevistas se llevaron a cabo en ocho ciudades metropolitanas de América Latina y España: San Salvador, El Salvador; San José, Costa Rica; Cali, Colombia; Caracas, Venezuela; Salvador de Bahía y Río de Janeiro, Brasil; Santiago, Chile, y Madrid, España.

Aunque la violencia asume diferentes formas y manifestaciones, la investigación se limitó al análisis de la violencia física interpersonal, entendida para fines del estudio como el uso intencional o la amenaza de usar la fuerza física que ejerce una persona para causar daño físico a otra, a un grupo de personas o al patrimonio personal o social. De acuerdo con los objetivos propuestos, la violencia interpersonal se evaluó a nivel de la pareja, de adultos hacia niños y de adultos hacia personas desconocidas. Los espacios evaluados se circunscribieron a los niveles doméstico, comunitario y social. Quedó excluida la evaluación de la violencia ejercida por instituciones y organizaciones de diferente índole, la violencia psicológica y la violencia sexual. Tampoco se consideraron las manifestaciones de violencia en el lugar de trabajo ni en las escuelas. 
FIGURA 1. Modelo para entender los procesos psicológicos y sociales que gobiernan la violencia

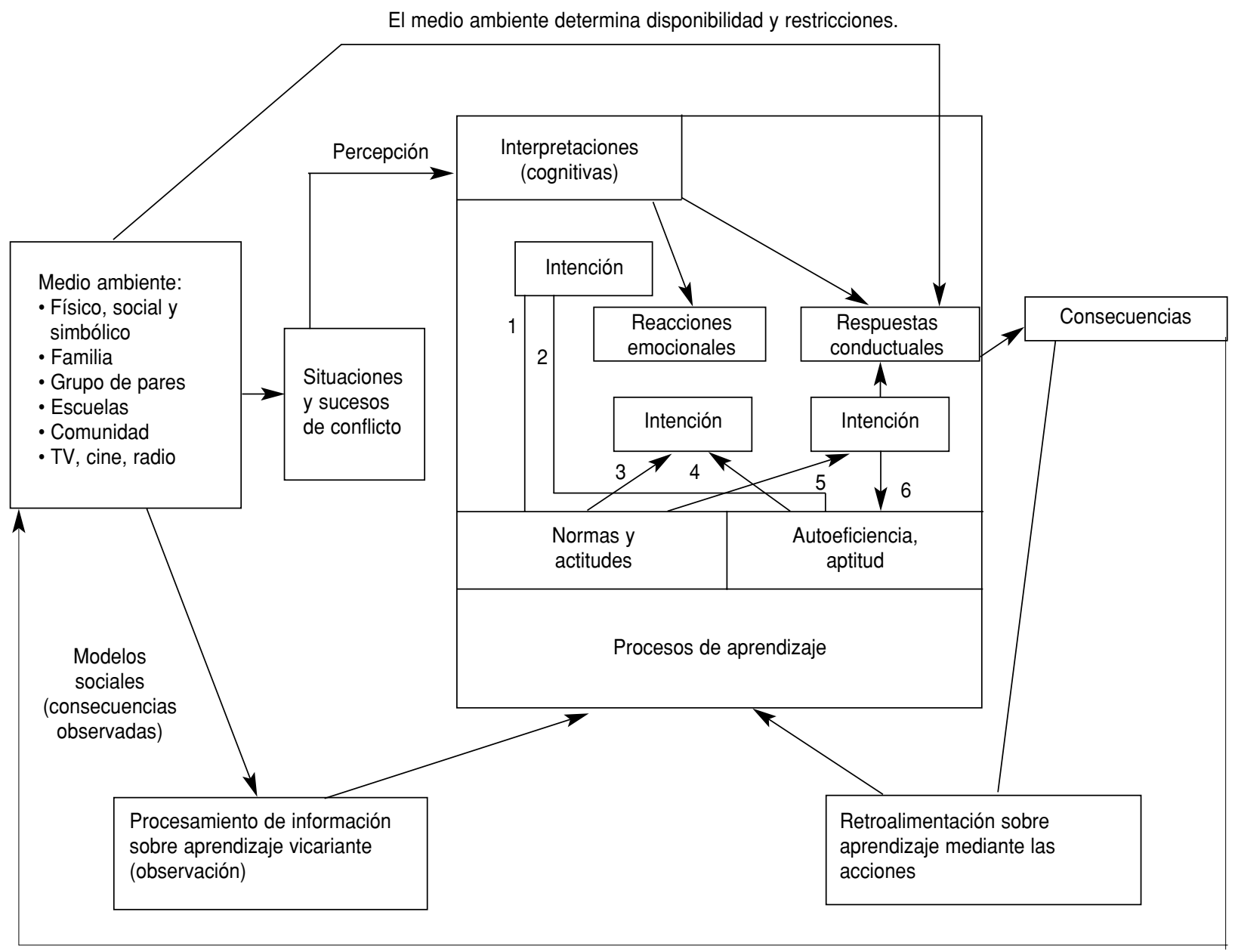

1 La interpretación de un conflicto depende de las creencias y valores que influyen en la atribución, por ejemplo, cuando una intención de provocación se infiere selectivamente a grupos.

2 La codificación de la información, la atribución y otras aptitudes cognitivas determinan cuán acertadamente se interpreta la situación de conflicto.

3 Dependiendo de las actitudes hacia la emoción y sus expresiones, algunas interpretaciones podrían desembocar en sentimientos de cólera y pesadumbre.

4 La aptitud de autocontrol cognitivo y emocional puede inhibir o aumentar la intensidad de la reacción y la posibilidad de responder.

5 Las respuestas preferidas se determinan mediante las normas y actitudes percibidas sobre su utilidad, propiedad, y demás.

6 La selección de la respuesta se hace según las aptitudes reales y la autoeficiencia percibida para realizar la conducta con efectividad y corrección.

Fuente: Ref. 8.

\section{El cuestionario ACTIVA}

En enero de 1996, el grupo de investigadores ${ }^{5}$ participó en un primer taller de trabajo auspiciado por la OPS y el

\footnotetext{
5 El estudio partió en la fase de planificación con la participación de 10 ciudades de América Latina y el Caribe (San Salvador, El Salvador; San José, Costa Rica; Cali, Colombia; Caracas y Maracaibo, Venezuela; Lima, Perú; Salvador de Bahía y Río de Janeiro, Brasil; Santiago, Chile y La Habana, Cuba); dos de Estados Unidos (Austin y Houston, Texas) y una de Canadá (Vancouver). En todas
}

Centro Colaborador de la OMS sobre Promoción de la Salud de la Universidad de Texas en Houston, con objeto de establecer el modelo descrito en la introducción, definir los constructos

estas ciudades se realizaron pruebas del cuestionario. Posteriormente, se incorporó al estudio la ciudad de Madrid, España. La encuesta solo se llevó a cabo con los métodos y la muestra total esperada en siete ciudades de América Latina y España. En Austin y Houston, Texas, se administró una versión resumida y modificada del cuestionario y la encuesta se realizó por teléfono. centrales, seleccionar indicadores para estos constructos, y definir los métodos empleados en el estudio. Partiendo de este modelo teórico y basándose en una combinación de elementos tomados de estudios anteriores, el equipo de investigadores identificó, adaptó y elaboró preguntas para el cuestionario ACTIVA. El cuestionario se diseñó con el fin de cuantificar variables psicosociales asociadas con la violencia, específicamente las 
CUADRO 1. Definición de conceptos del modelo para entender los procesos psicológicos y sociales que gobiernan la violencia. Proyecto ACTIVA, 1997

\begin{tabular}{|c|c|}
\hline Concepto & Definición \\
\hline Conflicto & Situaciones y sucesos que pueden provocar violencia debido a problemas en las relaciones entre individuos y grupos \\
\hline Interpretación & Proceso mediante el cual una persona que se encuentra en conflicto comprende determinados sucesos y situaciones \\
\hline Reacción & Consecuencia emocional de la interpretación del conflicto \\
\hline Respuesta & Comportamiento real que produce el conflicto, ya sea de tipo violento o no violento \\
\hline Normas & Reglas implícitas o explícitas de un grupo sobre los comportamientos, valores o creencias aceptados \\
\hline Actitudes & Evaluaciones relativamente permanentes, ya sean positivas o negativas, sobre personas, grupos, objetos o ideas \\
\hline Aptitud & Capacidad para hacer algo, lo que incluye operaciones cognitivas internas y capacidades conductuales relacionadas \\
\hline Autoeficacia & $\begin{array}{l}\text { Juicio sobre la capacidad personal para comportarse de cierta forma, para interpretar situaciones complejas o para controlar las } \\
\text { reacciones emocionales }\end{array}$ \\
\hline Intención & $\begin{array}{l}\text { Conocimiento sobre cómo se comportará una persona, cómo realizará una operación cognitiva determinada (interpretación), o cómo } \\
\text { experimentará o expresará una emoción en particular }\end{array}$ \\
\hline
\end{tabular}

normas, actitudes y habilidades asociadas con conductas de agresión y de victimización. Se identificaron variables que intervienen en la relación entre los componentes psicosociales y las conductas agresivas o de victimización, entre ellas, atributos sociodemográficos, condiciones socioeconómicas, características familiares, confianza en las instituciones, exposición a la televisión, embriaguez y tenencia de armas de fuego.

Para garantizar la utilización de un cuestionario estandarizado para los diferentes países participantes, así como para evaluar sus ventajas y limitaciones, se procedió a probar el cuestionario en tres pruebas piloto. Los investigadores alcanzaron un consenso con respecto a una primera versión del cuestionario en la reunión que se celebró en Houston. Este primer cuestionario se probó en las siguientes ciudades: Houston, San Salvador, San José, Cali, Caracas, Maracaibo, Río de Janeiro, Salvador de Bahía, Santiago y Vancouver. En cada ciudad se entrevistó a un total de 60 personas. Se seleccionaron 20 estudiantes universitarios, 20 personas de hogares de clase media o baja, y 20 pacientes que acudieron a consultas externas de hospitales públicos. En cada uno de estos tres grupos se trabajó con cuotas de edad y sexo.

Las bases de datos de todas las ciudades se combinaron en un solo archivo. Se calculó la distribución de frecuencias de cada pregunta para determinar el nivel de variabilidad logrado. Por añadidura, para cada una de las escalas se calcularon el grado de homogeneidad de los ítemes y el coeficiente de confiabilidad (alfa de Cronbach) de cada escala. Además de hacer el procesamiento estadístico de la información recolectada, los investigadores de cada ciudad escrutaron los resultados con los entrevistadores y, en algunos casos, constituyeron grupos focales con expertos.

Con la información de esta primera aplicación se celebró la segunda reunión en San José, Costa Rica, en la cual asesores e investigadores de los países participantes debatieron durante una semana los alcances de los resultados e introdujeron los cambios necesarios. Como resultado de los análisis de la primera versión del cuestionario se realizaron cambios sustanciales. Específicamente, se redactaron de nuevo algunas de las preguntas con el propósito de garantizar un equilibrio entre los contenidos de cada escala con respecto a situaciones personales, comunitarias e institucionales, así como para garantizar mayor coherencia conceptual. Además, se modificó el orden de las secciones, para dejar para el final aquellas que se referían a conductas directas del entrevistado, por considerarlas más amenazantes para la persona. En función de los análisis realizados y del modelo teórico de base, se procedió a reestructurar un conjunto diferente de escalas, combinando muchos ítemes originales con nuevos elementos redactados por el equipo de investigadores. Por último, se redujo el número de preguntas de la mayoría de las escalas a fin de reducir la duración de la administración de los cuestionarios.
Estas transformaciones obligaron a desarrollar una segunda prueba piloto que se realizó en San Salvador, San José, Río de Janeiro y Lima. En cada ciudad se escogieron ocho segmentos censales al azar y en cada uno de ellos se entrevistó a 10 personas, una por vivienda, utilizando cuotas de sexo y edad. Al igual que en la primera etapa, se calculó la distribución de frecuencias para los ítemes, el nivel de homogeneidad y la confiabilidad de las escalas. Puesto que el tamaño de la mayoría de las escalas se redujo, y con el fin de hacer comparaciones con los resultados obtenidos en la primera etapa, los índices de confiabilidad se ajustaron mediante la fórmula de la profecía de Spearman-Brown (9). En esta segunda versión, las escalas mostraron una mejor consistencia interna, a excepción de las escalas de habilidades y violencia hacia los niños, por lo que en estos dos casos se decidió retornar a la estructura original.

En una nueva reunión, también celebrada en San José, se elaboró una tercera versión con algunas modificaciones en cuanto a la estructura de algunas de las escalas. El tercer cuestionario se puso a disposición de todos los investigadores y se organizó un proceso de análisis y debate por correo electrónico. En esta etapa el proceso se centró especialmente en el ordenamiento de las escalas del cuestionario, la conformación final de cada sección y la redacción de los ítemes, especialmente en lo que respecta a su corrección gramatical y filológica. En cada ciudad se elaboró una lista de propuestas y sugerencias, que, 
a su vez, se organizaron en una tabla resumen, ordenadas por escalas y por preguntas. El resumen fue entonces sometido a la consideración de cada investigador y, por consenso, se definió la versión número cuatro y definitiva del cuestionario.

Los indicadores y la fuente utilizada para elaborar los indicadores, así como el cuestionario con las preguntas comunes para todos los países, se encuentran en los apéndices 2 y 3 de esta monografía, respectivamente. La versión definitiva del cuestionario quedó estructurada en 15 dimensiones, que se describen a continuación.

1. Atributos personales, socioeconómicos y familiares (DE). Se incluyeron preguntas relacionadas con las características del entrevistado y su familia. En concreto, la estructura familiar se estableció considerando el número de miembros y su posición dentro del núcleo familiar, así como el parentesco con el jefe de hogar, el sexo, la edad, el nivel educativo y la percepción de ingresos por trabajo de cada uno de los miembros. Se evaluaron asimismo el grado de hacinamiento y la asistencia a la escuela de los niños menores de 12 años. En cuanto a las características del entrevistado, se obtuvo información con respecto a la edad, el estado civil, la situación laboral, la nacionalidad, la religión y la raza. En esta sección se incluyeron las siguientes variables conductuales: exposición a la televisión, gusto por programas violentos y frecuencia de embriaguez. La pregunta sobre embriaguez se basó en una encuesta del Centro para el Control y la Prevención de Enfermedades (CDC) de los Estados Unidos de América (10).

2. Violencia física y castigo corporal de menores (NI). Se indagó la responsabilidad del entrevistado en el cuidado de sus hijos o de otros niños. $\mathrm{Si}$ el entrevistado era responsable de niños entre 2 y 15 años, se evaluó la frecuencia de distintas prácticas disciplinarias y castigos. Cuando el entrevistado tenía más de un niño a su cargo, se escogió aquel que hubiese cumplido años más recientemente. La escala con que se evaluaron las conductas agresivas de los padres hacia los hijos estuvo constituida por tres preguntas (gritar con rabia, pegar una palmada, pegar con un objeto duro). Estas preguntas se basaron en el conflict tactic scale, escala elaborada por Straus (11). Con una cuarta pregunta se estimó la frecuencia con que se aplica un método de disciplina no violento.

3. Violencia entre parejas (PA). Aquellos entrevistados que vivían en el momento de la entrevista con su pareja respondieron a tres preguntas referentes a la frecuencia con que habían agredido a la pareja y a tres preguntas sobre la frecuencia con que fueron víctimas de agresión por la pareja (gritar, pegar una cachetada, pegar con un objeto duro). Estas preguntas también se basaron en la escala elaborada por Straus (11).

4. Otras conductas violentas hacia personas fuera de la familia (OC). Por medio de cuatro preguntas, basadas en la escala de Straus (11), se evaluó la frecuencia con que el entrevistado había agredido a personas fuera del núcleo familiar.

5. Normas sociales y culturales (NO). El nivel de aceptación o rechazo de diferentes normas sociales asociadas con el uso de la violencia contra la pareja y contra los niños, así como la aceptación de la violencia o ilegalidad en la sociedad, se evaluaron mediante 12 preguntas, algunas de las cuales se basaron en el estudio de Cohen y Nisbett (12).

6. Habilidades (HA). Para evaluar el grado de autoeficacia que manifestó poseer el entrevistado para resolver conflictos de forma no violenta en situaciones específicas se emplearon cinco preguntas.

7. Actitudes de aprobación de la violencia (AC). Mediante 11 preguntas se evaluó la aprobación de conductas violentas específicas. Esta dimensión se estructuró en dos partes: con cinco preguntas se evaluaron el grado de aprobación y compren- sión de conductas violentas de una tercera persona y con seis, el grado de acuerdo o desacuerdo con conductas violentas específicas.

8. Relaciones intergrupales (RI). Para evaluar las tendencias hacia la discriminación por clase social, religión, grupo étnico e ideas políticas se formularon cuatro preguntas. Con una de ellas se evaluó el grado de acuerdo con el derecho a sacar a ciertos grupos del vecindario.

9. Confianza en instituciones (IN). Por medio de siete preguntas se evaluó el nivel de eficiencia percibido con respecto a siete diferentes instituciones relacionadas con la justicia criminal y los medios de comunicación. Como base se utilizó la Encuesta Social General de los Estados Unidos (13).

10. Actitudes sociales (AS). Las actitudes hacia el sistema social y el nivel de anomia del entrevistado se evaluaron mediante cinco preguntas, utilizando como base el Latinobarómetro (14).

11. Aceptación del uso institucional de la fuerza (RN). Con tres preguntas se evaluó el grado de aceptación o rechazo de medidas gubernamentales violentas contra otros países o contra grupos internos, y si la presencia militar en las calles es necesaria.

12. Normas familiares (NF). Las normas familiares y, en concreto, si el castigo corporal se usó contra el entrevistado en su infancia se evaluaron mediante dos preguntas.

13. Uso de armas (AR). La utilización de armas de fuego en el hogar y fuera de este, así como el deseo de tenerlas, se evaluaron con cuatro preguntas.

14. Victimización (VI). Para evaluar experiencias de victimización o de violencia del entrevistado, desde robo a mano armada hasta suicidio $\mathrm{u}$ homicidio de un familiar, se formularon 11 preguntas.

15. Efectos de la violencia (OP). Por último, se formularon nueve preguntas adicionales para evaluar la sensación de inseguridad y miedo que percibe el entrevistado en su comunidad y el cambio en las 
prácticas cotidianas de vida como consecuencia de la violencia. La utilización de estas preguntas se dejó a la discreción de los investigadores de cada ciudad que participó en el estudio.

\section{Área geográfica de interés, población del estudio y tamaño de la muestra}

El análisis realizado en el estudio se concentró en el fenómeno de la violencia interpersonal urbana. La población de interés estuvo comprendida por hombres y mujeres, nacionales y extranjeros, que fuesen residentes habituales en los hogares particulares de las áreas metropolitanas de las ciudades seleccionadas. En algunas ciudades la solicitud de consentimiento e información de los padres o tutores de menores fue un requisito legal. Por tal motivo y para evitar sesgos en las respuestas, se optó por establecer como límite inferior de edad los 18 años. Por razones de acceso y condiciones de salud, se acordó fijar el límite superior de la edad del entrevistado en 70 años, aunque cabe señalar que en la población de interés de la ciudad de Cali se incluyó a personas desde los 15 años de edad cumplidos, y en la ciudad de Madrid no se definió un límite superior de edad.

Los criterios para definir el tamaño de la muestra se establecieron con el objeto de conseguir una confianza en la estimación de proporciones poblacionales de $95 \%$ o, en otras palabras, de limitar el error de estimación a 5\%. Para realizar este cálculo se aceptó un efecto de diseño igual a 3. El tamaño de muestra necesario fue de 1200 personas para cada ciudad. Dado que se previó un diseño sin sustitución, se aceptó como porcentaje de falta de respuesta el comprendido entre 25 y $35 \%$, sobre todo en los estratos medios y altos.

\section{Selección de la muestra}

La muestra fue seleccionada mediante un muestreo multietápico, estratificado y por conglomerados, proporcional según la situación socioeconómica y la densidad de población y sin reemplazo o sustitución. Este proceso de selección fue seguido en todas las ciudades con excepción de Cali, razón por la cual se realizaron ajustes en la muestra de esta ciudad. En el apéndice 1 de esta publicación se presentan los cuadros donde se resume la información principal del muestreo seguido en cada ciudad.

Aunque el muestreo varió en algunas ciudades, en la mayoría de ellas la selección se hizo en las siguientes etapas. Primero, se seleccionaron las unidades más pequeñas de la división politicoadministrativa de la ciudad. Para ello, se definió el número de unidades de la división politicoadministrativa de la región metropolitana que deberían ser incluidas en la muestra. Se estipuló que el número de unidades no podría ser menor de $50 \%$ de todas las unidades. A las dos unidades de mayor población se les asignó siempre una probabilidad de selección igual a 1. Segundo, con los segmentos censales de las unidades seleccionadas se conformaron, como mínimo, tres estratos socioeconómicos (bajo, medio y alto). Para determinar la clasificación de los segmentos por estratos, se siguieron los criterios establecidos por las direcciones de estadísticas y censos. Los criterios utilizados por cada país fueron diferentes y la estratificación a priori no se llevó a cabo en todas las ciudades. En el cuadro 2 se resumen los criterios y técnicas utilizadas en cada ciudad. Tercero, en cada estrato se seleccionaron aleatoriamente los segmentos censales con peso proporcional a la población (proportional population selection o PPS). Cuarto, se seleccionaron $10 \%$ de los hogares con arreglo al número total de viviendas en el segmento. De acuerdo con la descripción cartográfica de Estadísticas y Censos, se seleccionaron las viviendas mediante muestreo aleatorio simple o sistemático y, preferiblemente, por muestreo sistemático. Cada hogar de las viviendas seleccionadas se debía contactar. Quinto, se seleccionaron las personas en el hogar. En esta etapa hubo variantes según las ciudades. En algunas (San José, San Salvador, Santiago) se siguió un muestreo sistemático de los renglones de la tabla de miembros del hogar según el procedimiento establecido en el protocolo (8). En otras se seleccionó en cada hogar a una persona entre las edades comprendidas mediante un muestreo aleatorio simple. En la ciudad de Río de Janeiro se censó a todas las personas entre los 18 y 70 años residentes en los hogares particulares seleccionados y luego se procedió a seleccionarlas al azar.

La muestra final estuvo compuesta por 10821 personas. En general, los porcentajes de respuesta se encontraron comprendidos entre los límites establecidos salvo algunas excepciones. En San José, Río de Janeiro y Madrid el tamaño de la muestra final fue ligeramente menor del esperado. Se registraron mayores rechazos en adultos jóvenes varones de los estratos altos, por lo cual en las muestras se observa una sobrerrepresentación de mujeres con predominio de los estratos medio y bajo (cuadro 3). Se decidió no ajustar la muestra por sesgo de selección, dado que se consideró cuestionable aceptar como válido el supuesto implícito de dicho ajuste, es decir, dar por hecho que las personas que no contestaron se comportarían y contestarían igual a las personas que sí contestaron.

\section{Recolección y procesamiento de datos}

La recolección de la información varió de ciudad en ciudad. Se inició en Cali en julio de 1996 y finalizó en Santiago en marzo de 1997. La duración media del trabajo de campo fue de aproximadamente 8 semanas.

En todas las ciudades se trabajó con entrevistadores capacitados para realizar las encuestas a domicilio y para el manejo ético y técnico de las entrevistas. Cada equipo fue sometido a un proceso cuidadoso de capacitación, que incluyó el análisis y el consenso de los objetivos generales del estudio, una revisión de cada elemento del cuestionario y una revisión del procedimiento de selección de los entrevistados en cada hogar. Se preparó además una guía del entrevistador 
CUADRO 2. Marcos muestrales, definición de unidades de selección y criterios de estratificación socioeconómica por ciudad. Proyecto ACTIVA, 1997

\begin{tabular}{|c|c|c|c|}
\hline $\begin{array}{c}\text { Ciudad } \\
\text { (Área geográfica) }\end{array}$ & $\begin{array}{l}\text { Marco } \\
\text { muestral }\end{array}$ & $\begin{array}{l}\text { Unidad primaria } \\
\text { de muestreo }\end{array}$ & Estratificación socioeconómica, criterios y procedimientos \\
\hline $\begin{array}{l}\text { Salvador, Bahía } \\
\text { (Región Metropolitana) }\end{array}$ & $\begin{array}{l}\text { Censo Demográfico de } \\
\text { Brasil (1991) }\end{array}$ & $\begin{array}{l}\text { Segmentos censales } \\
\text { agrupados en áreas }\end{array}$ & $\begin{array}{l}\text { Renta de los domicilios (estrato alto: más de } 50 \% \text { de los hogares } \\
\text { con ingreso > } 5 \text { salarios mínimos; estrato bajo: más de } 50 \% \text { de } \\
\text { los hogares con ingreso } \leq 2 \text { salarios mínimos; estrato medio: } \\
\text { mezcla de ingresos altos y bajos) }\end{array}$ \\
\hline $\begin{array}{l}\text { Cali } \\
\text { (Ciudad de Cali) }\end{array}$ & $\begin{array}{l}\text { Cartografía censal del } \\
\text { Departamento Nacional } \\
\text { de Estadística (1993) }\end{array}$ & Manzanas & $\begin{array}{l}\text { Postestratificación a partir del índice de clasificación de la } \\
\text { Dirección Nacional de Estadísticas (DANE). No definió estratos } \\
\text { para la selección. }\end{array}$ \\
\hline $\begin{array}{l}\text { Caracas } \\
\text { (Área Metropolitana) }\end{array}$ & $\begin{array}{l}\text { Muestra Maestra de } \\
\text { Viviendas de Venezuela }\end{array}$ & Segmento censal & $\begin{array}{l}\text { Indicador de Necesidades Básicas Insatisfechas, con } \\
\text { postestratificación }\end{array}$ \\
\hline $\begin{array}{l}\text { Madrid } \\
\text { (Municipalidad de Madrid) }\end{array}$ & $\begin{array}{l}\text { Censo de Población del } \\
\text { Ayuntamiento de Madrid } \\
(1996)\end{array}$ & Segmento censal & $\begin{array}{l}\text { Índice del Departamento de Estadísticas del Ayuntamiento de } \\
\text { Madrid compuesto por desempleo, ocupación, escolaridad, } \\
\text { categoría profesional, valor del suelo y vehículos. }\end{array}$ \\
\hline $\begin{array}{l}\text { Río de Janeiro } \\
\text { (Región Metropolitana) }\end{array}$ & $\begin{array}{l}\text { Censo Demográfico de } \\
\text { Brasil (1991) }\end{array}$ & Segmento censal & $\begin{array}{l}\text { Renta del jefe del hogar, promedio de años de educación del jefe } \\
\text { de hogar y hacinamiento (estrato alto: renta promedio }=6,1 \\
\text { salarios mínimos; estrato medio: } 4,4 \text { salarios mínimos; estrato } \\
\text { bajo: } 1,9 \text { salarios mínimos) }\end{array}$ \\
\hline $\begin{array}{l}\text { San José } \\
\text { (Región Metropolitana) }\end{array}$ & $\begin{array}{l}\text { Censo de Población } \\
\text { (1984) y proyecciones del } \\
\text { CELADE (1995) }\end{array}$ & Segmento censal & $\begin{array}{l}\text { Regresión multivariante con tenencia de artefactos eléctricos, } \\
\text { ocupación y escolaridad del jefe. }\end{array}$ \\
\hline $\begin{array}{l}\text { San Salvador } \\
\text { (Área Metropolitana) }\end{array}$ & V Censo de Población & Segmento censal & $\begin{array}{l}\text { Indicador de Necesidades Básicas Insatisfechas. } \\
\text { Postestratificación }\end{array}$ \\
\hline $\begin{array}{l}\text { Santiago } \\
\text { (Gran Santiago) }\end{array}$ & $\begin{array}{l}\text { Censo de Población y } \\
\text { Vivienda (1992) }\end{array}$ & Segmento censal & $\begin{array}{l}\text { Escolaridad, ocupación, calidad de la vivienda, hacinamiento, y } \\
\text { equipamiento de la vivienda. }\end{array}$ \\
\hline
\end{tabular}

Fuente: Informes sobre la muestra de cada ciudad.

que incluía por escrito una descripción detallada del cuestionario, del procedimiento de selección y de los mecanismos idóneos para abordar la resistencia y el rechazo parcial o total (15). Esta guía se utilizó en todas las ciudades.

A cada participante se le explicó el motivo de la encuesta y la forma como se iban a utilizar los datos. También se le explicó que la encuesta era anónima, que la participación debía ser voluntaria, y que podían negarse a responder cualquier pregunta o detener la entrevista en el momento que lo deseara. Se obtuvo el consentimiento verbal de cada entrevistado. Cuando los investigadores identificaron situaciones de riesgo, de posible maltrato o de abuso infantil o de otro miembro de la familia, se procedió a asesorar a las víctimas sobre mecanismos y opciones de atención. Además, estas personas fueron remitidas a personal profesional especializado.
El proceso de recolección tuvo dos etapas de supervisión. La primera se realizó directamente en el campo, donde personal especializado coordinó, revisó y orientó el trabajo en el lugar de la obtención de datos. La segunda se llevó a cabo en las respectivas oficinas, donde cada cuestionario completo fue sometido a una revisión rigurosa para garantizar que estuviese totalmente contestado, que el proceso de selección hubiese sido adecuado y que las respuestas contaran con la coherencia necesaria. Para ambas etapas se elaboró una guía escrita destinada a orientar el trabajo de supervisión (15).

Sobre la base de un programa establecido para tales fines, cada investigador construyó su base de datos para cada ciudad y realizó un análisis de incongruencias y la correspondiente limpieza de datos. Posteriormente, todas las bases de datos se compilaron en una sola base regional con las variables comunes a todas las ciudades.
Bajo la coordinación de la OPS se hizo un trabajo minucioso de revisión y limpieza de la base de datos regional en el cual participaron todos los investigadores. Los análisis de los resultados y los datos que se consignan en esta publicación se elaboraron a partir de la base de datos compilada ACTIVA (16).

\section{ALCANCE Y LIMITACIONES}

El proyecto ACTIVA es un estudio transversal que permitirá establecer una línea de base para evaluar futuros proyectos de prevención de la violencia y analizar asociaciones entre las conductas violentas y otros tipos de variables y dimensiones socioculturales y ambientales. Los métodos empleados en este estudio han permitido construir diversas escalas e indicadores que podrán utilizarse en otras investigaciones en el área. El haber sido probados en 
CUADRO 3. Descripción de la muestra por ciudad. Proyecto ACTIVA, 1997

\begin{tabular}{|c|c|c|c|c|c|c|c|c|c|c|c|c|c|c|c|c|}
\hline & \multicolumn{2}{|c|}{$\begin{array}{c}\text { Salvador, } \\
\text { Bahía, } \\
\text { Brasil }\end{array}$} & \multicolumn{2}{|c|}{$\begin{array}{c}\text { Cali, } \\
\text { Colombia }\end{array}$} & \multicolumn{2}{|c|}{$\begin{array}{l}\text { Caracas, } \\
\text { Venezuela }\end{array}$} & \multicolumn{2}{|c|}{$\begin{array}{l}\text { Madrid, } \\
\text { España }\end{array}$} & \multicolumn{2}{|c|}{$\begin{array}{c}\text { Rio de } \\
\text { Janeiro, } \\
\text { Brasil }\end{array}$} & \multicolumn{2}{|c|}{$\begin{array}{c}\text { San } \\
\text { José, } \\
\text { Costa Rica }\end{array}$} & \multicolumn{2}{|c|}{$\begin{array}{c}\text { San } \\
\text { Salvador, } \\
\text { El Salvador }\end{array}$} & \multicolumn{2}{|c|}{$\begin{array}{l}\text { Santiago, } \\
\text { Chile }\end{array}$} \\
\hline & $n$ & $\%$ & $n$ & $\%$ & $n$ & $\%$ & $n$ & $\%$ & $n$ & $\%$ & $n$ & $\%$ & $n$ & $\%$ & $n$ & $\%$ \\
\hline & \multicolumn{13}{|c|}{ Sexo } & 81 & 1212 & 86 \\
\hline Hombres & 633 & 45,7 & 1061 & 46,4 & 506 & 39,0 & 430 & 38,9 & 484 & 43,4 & 479 & 42,4 & 576 & 44,7 & 567 & 46,8 \\
\hline $18-24$ & 311 & 22,5 & 516 & 22,5 & 281 & 21,7 & 221 & 20,0 & 202 & 18,1 & 227 & 20,1 & 268 & 20,8 & 247 & 20,4 \\
\hline $25-44$ & 713 & 51,5 & 1205 & 52,6 & 642 & 49,5 & 479 & 43,3 & 523 & 46,9 & 492 & 43,5 & 646 & 50,1 & 602 & 49,7 \\
\hline $45-64$ & 322 & 23,3 & 493 & 21,5 & 312 & 24,1 & 318 & 28,8 & 326 & 29,3 & 329 & 29,1 & 318 & 24,7 & 301 & 24,8 \\
\hline $65-70$ & 38 & 2,7 & 75 & 3,3 & 62 & 4,8 & 87 & 7,9 & 63 & 5,7 & 83 & 7,3 & 58 & 4,5 & 62 & 5,1 \\
\hline \multicolumn{17}{|l|}{ Estrato social } \\
\hline Alto & 113 & 8,2 & 221 & 9,9 & 30 & 2,3 & 219 & 19,8 & 161 & 14,5 & 154 & 13,6 & 163 & 12,6 & 168 & 13,9 \\
\hline Medio & 507 & 36,6 & 926 & 41,5 & 314 & 24,2 & 661 & 59,8 & 303 & 27,2 & 577 & 51,0 & 457 & 35,4 & 349 & 28,8 \\
\hline Secundaria & 609 & 44,8 & 1204 & 53,4 & 548 & 42,3 & 359 & 32,7 & 241 & 21,7 & 388 & 34,3 & 417 & 32,7 & 623 & 51,4 \\
\hline Universitaria o técnica & 259 & 19,0 & 324 & 14,4 & 507 & 39,1 & 332 & 30,2 & 206 & 18,6 & 408 & 36,1 & 342 & 26,8 & 350 & 28,9 \\
\hline \multicolumn{17}{|l|}{ Estado civil } \\
\hline Soltero & 516 & 37,3 & 718 & 31,4 & 419 & 32,3 & 408 & 37,0 & 311 & 27,9 & 325 & 28,7 & 351 & 27,2 & 320 & 26,4 \\
\hline Casado & 466 & 33,7 & 695 & 30,4 & 544 & 42,0 & 584 & 52,9 & 544 & 48,8 & 590 & 52,2 & 558 & 43,3 & 722 & 59,6 \\
\hline Unión libre & 204 & 14,8 & 643 & 28,1 & 142 & 11,0 & 27 & 2,4 & 127 & 11,4 & 75 & 6,6 & 261 & 20,2 & 63 & 5,2 \\
\hline Separado/divorciado & 117 & 8,5 & 145 & 6,3 & 154 & 11,9 & 40 & 3,6 & 77 & 6,9 & 91 & 8,0 & 75 & 5,8 & 63 & 5,2 \\
\hline Viudo & 80 & 5,8 & 86 & 3,8 & 37 & 2,9 & 45 & 4,1 & 55 & 4,9 & 50 & 4,4 & 45 & 3,5 & 44 & 3,6 \\
\hline \multicolumn{17}{|l|}{ Situación laboral } \\
\hline Ocupado & 803 & 59,9 & 1355 & 59,2 & 819 & 63,1 & 487 & 46,5 & 678 & 61,2 & 595 & 52,6 & 889 & 69,0 & 646 & 53,3 \\
\hline Desocupadoa & 159 & 11,9 & 205 & 9,0 & 117 & 9,0 & 156 & 14,9 & 95 & 8,6 & 45 & 4,0 & 69 & 5,4 & 66 & 5,4 \\
\hline Estudiante & 79 & 5,9 & 87 & 3,8 & 66 & 5,1 & 133 & 12,7 & 32 & 2,9 & 95 & 8,4 & 66 & 5,1 & 126 & 10,4 \\
\hline Pensionado & 134 & 10,0 & 61 & 2,7 & 0 & 0,0 & 98 & 9,4 & 93 & 8,4 & 113 & 10,0 & 58 & 4,5 & 71 & 5,9 \\
\hline
\end{tabular}

a Desocupado: Persona que no trabaja y que no ha buscado ocupación.

diferentes países facilita su aplicación en otras zonas de América Latina. Se consideraron indicadores familiares y de los hogares que permitirán introducir algunas dimensiones del contexto y superar en parte los análisis de los estudios tradicionales con datos exclusivamente individuales.

Es importante resaltar los alcances análiticos de este estudio. La rigurosidad en el diseño del instrumento, en la obtención de la muestra y en el proceso de obtención de la información, así como el hecho de que se haya utilizado un método estándar en diferen- tes ciudades, posibilita no solo el estudio individual del fenómeno de la violencia en cada ciudad, sino sobre todo la comparación de los resultados entre los diferentes centros urbanos. La variabilidad de la intensidad con la que se presenta la violencia en distintas ciudades permite llevar a cabo comparaciones de enorme interés análitico y conceptual. El análisis comparativo entre ciudades constituye una aportación fundamental para entender las dimensiones actitudinales, conductuales y culturales que prevalecen en los medios urbanos y brinda mayores y mejo- res pruebas para el establecimiento de acciones e intervenciones de corto y mediano plazo.

Los métodos empleados en el proyecto ACTIVA presentan algunas limitaciones. Primero, el estudio permite extraer conclusiones sobre las conductas, actitudes y otras variables notificadas por los entrevistados que pueden no reflejar las prevalencias reales en la población. Las magnitudes y los niveles de las conductas determinadas mediante la encuesta fueron las notificadas por los entrevistados y, por tanto, han de considerarse como estimaciones 
de parámetros de referencia y no como prevalencias reales en la población. Aunque se procuró controlar el efecto de la deseabilidad social en las respuestas, las características del tema investigado hacen suponer que debido a este efecto se pudo subestimar la dimensión del problema. Segundo, todos los estudios tuvieron diseños muestrales similares, aunque no se aplicaron en todos las mismas fases. Además, la selección tanto de las unidades intermedias como finales del muestreo se realizó siguiendo principios probabilísticos. Sin embargo, en la conformación de la muestra final se introdujeron sesgos de selección como resultado de la falta de respuesta, que fue selectiva por estrato socioeconómico, edad y sexo. Este problema no puede resolverse mediante ajustes estadísticos y es preciso tenerlo en cuenta al realizar los análisis y extraer las conclusiones.

Tercero, no se pueden generalizar los resultados del proyecto ACTIVA a toda la Región. El estudio no pretende obtener una representatividad de toda América Latina. Las ciudades que intervinieron lo hicieron exclusivamente por disponibilidad y posibilidades materiales $y$, por consiguiente, la muestra regional ha de considerarse estrictamente intencional. Del mismo modo, el sumar los datos de las ocho ciudades para establecer resultados generales puede tener consecuencias metodológicas pe- ligrosas, puesto que el peso relativo de cada una de las muestras no representa las enormes diferencias poblacionales entre las ciudades participantes. Es decir, los datos pueden combinarse con fines comparativos, pero no con fines de generalización.

Finalmente, cabe advertir que con el método propuesto es posible analizar la asociación entre las conductas violentas y diferentes factores de riesgo y atributos a nivel individual en un momento en el tiempo. Sin embargo, este diseño tiene limitaciones para establecer relaciones causales y no permite evaluar el contexto en que la violencia se manifiesta y desarrolla en cada situación particular.

\section{REFERENCIAS}

1. Pan American Health Organization. Violence: a growing public health problem in the Region. Epidemiol Bull 1990;11:1-6.

2. Levav I, Guerrero R., Phebo L, et al. A regional effort to reduce corporal punishment in children. The promotion of a community program through primary care. Washington, D.C.: Pan American Health Organization/ World Health Organization; 1995. (Document $\mathrm{PAHO} / \mathrm{WHO} / \mathrm{HPP} / 45.95$.)

3. Hartigan P. La OPS enfoca el problema de la violencia contra la mujer. Rev Panam Salud Pública 1999;2:290-294.

4. Bandura A. Aggression: a social learning analysis. Englewood Cliffs, N.J.: PrenticeHall; 1973.

5. Bandura A. Social foundations of thought and action: a social cognitive theory. Englewood Cliffs, N.J.: Prentice-Hall; 1986.

6. Bandura A. Social cognitive theory of moral thought and action. En: Kurtines WM, Gewirtz JL, eds. Handbook of moral behavior and development. Hillsdale, N.J.: Lawrence Erlbaum; 1991;1:45-103

7. McAlister A, Orlandi M, Puska P, Zbylot P, Bye LL. Behavior modification in public health: principles and illustrations. En: Holland WW, Detels R, Knox EG, eds. Oxford Textbook of Public Health. 2nd ed. Oxford: Oxford Medical Publications; 1991.

8. McAlister A, Vélez LF, De los Ríos R, Fournier M, Piquet-Carneiro L. Protocolo del Estudio Multicéntrico "Actitudes y Normas Culturales hacia la Violencia en Ciudades Seleccionadas de América Latina y España". Proyecto ACTIVA. Washington, D.C.: Organización Panamericana de la Salud, Coordinación de Investigaciones; 1999. (Serie Investigaciones en Salud Pública, Documentos Técnicos No. 1.)

9. DeVellis RF. Scale Development: theory and applications. Newbury Park, Ca.: Sage Publications.

10. Centers for Disease Control and Prevention. State and sex-specific prevalence of selected characteristics-behavioral risk factor surveillance system, 1994 and 1995. MMWR 1997; 46:1-31.

11. Straus MA. Measuring intrafamily conflict and violence: the conflict tactic (CT) scales. En: Straus MA, Gelles RJ, eds. Physical violence in American families: risk factors and adapta- tions to violence in 8,145 families. New Brunswick, N.J.: Transaction Publications; 1990.

12. Cohen D, Nisbett RE. Self-protection and the culture of honor: explaining southern violence. Personality Soc Psychol Bull 1994; 20:551-567.

13. National Opinion Research Center. General Social Surveys. Cummulative Codebook 1972-1994. Codebook prepared by NORC ed. Chicago: Distributed by the Roper Public Opinion Research Center; 1994.

14. Turner FC, Martz JD. Institutional confidence and democratic consolidation in Latin America. Studies in Comparative International Development 1997;32:65-84.

15. Organización Panamericana de la Salud, Coordinación de Investigaciones. Proyecto ACTIVA. Manual del entrevistador, del supervisor de campo y del supervisor de oficina. Washington, D.C.: OPS; 1999. (Serie Investigaciones en Salud Pública, Documento Técnico No. 2.)

16. Organización Panamericana de la Salud, Coordinación de Investigaciones. Proyecto ACTIVA. Base de datos ACTIVA. Washington, D.C.: OPS; 1999. (Serie Investigaciones en Salud Pública, Documento Técnico No. 5.) 
ABSTRACT The purpose of this article is to describe the conceptual bases and methods used in the ACTIVA multicenter study, as well as the process employed in the different stages of the research. General information is given on the approach, design, and methods that were applied in the cities that participated in the study: San Salvador, El Salvador; San José, Costa Rica; Cali, Colombia; Caracas, Venezuela; Salvador, Bahia, Brazil; Rio de Janeiro, Brazil; Santiago, Chile; and Madrid, Spain. The objectives of the study were to: (1) analyze and compare among cities the prevalence of verbal and physical aggressive behaviors toward children, the spouse or other partner, and persons outside the family; and (2) identify personal, environmental, and socioeconomic factors associated with these violent behaviors. The purpose of the ACTIVA project was to generate information to help guide violence-prevention policies and programs in the cities and to provide criteria for decision-making, as well as to obtain baseline data to use in evaluating prevention policies and programs. To achieve the proposed objectives, a single, common questionnaire was prepared and validated. The questionnaire was used in face-to-face interviews with residents of private urban homes who were between 18 and 70 years old. The sample was selected in each city through stratified multistage sampling of clusters proportionate to the socioeconomic strata of the general population and without any substitutions. The final sample consisted of 10821 people. In general, the response rates were within the established limits. Exceptions included San José, Rio de Janeiro, and Madrid, where the size of the final sample was slightly smaller than anticipated. More young men of higher socioeconomic strata declined to participate, so that the samples had an overrepresentation of women from medium and lower strata. The article concludes with a description of the scope and limitations of the study, in terms of both the approach and methods. With their comparative analyses, studies of this type can contribute to identifying the differences and similarities between cities. The article shows that with this method it is possible to analyze how violent behaviors are associated with different risk factors and individuals' characteristics, at a point in time. However, this design is limited in terms of establishing causal relationships, and it did not allow an evaluation of the context of every specific instance of violence. 